\title{
Sensory characteristics of wholegrain
}

\begin{abstract}
Wholegrain foods are known to be health-beneficial but their sensory characteristics may be a limiting factor for consumption. Many cereals like rye, oats, barley and sorghum are actually used mainly as wholegrain, whereas for wheat the situation is the opposite. This review deals with factors that differentiate the sensory properties of wholegrain and branrich foods from those of refined cereal foods. Hitherto established means of modulating sensory quality and thus improving acceptability of wholegrain foods are also presented.
\end{abstract}

Volume 10 Issue $6-2020$

Tanya Bhasin

MSc Food \& Nutrition, India

Correspondence: Tanya Bhasin, MSc Food \& Nutrition, India, Email bhasintanya99@gmail.com

Received: February 29, 2020 | Published: December 29, 2020

\section{Introduction}

Cereal grains are a major energy source globally. In Europe, wheat is the most commonly used of all grains. While bread and pasta are elementary parts of the daily diet, the consumption of biscuits, breakfast cereals and snacks is increasing. In the past, cereal foods were based on wholegrain flour. Industrialization brought along development of the milling industry, which led to increased production of refined white flour and separation of the outer bran layers of the kernel mainly to be used as animal feed or biofuel. ${ }^{1-5}$

This refining, driven by facilitated processing, food safety and consumer preferences for white flour, was accompanied by a decrease in intake of dietary fibre (DF) and nutrients present in the wholegrain. Due to growing prosperity and abundant availability of food, white wheat flour became the standard raw material for the wheat based cereal industry, leading to product and process development of refined wheat products. In addition to improving public health, utilisation of wholegrains for human food would also contribute to food security and sustainable food production. Identification of factors underlying flavour and texture formation helps to improve the sensory attributes of cereal products containing wholegrain or bran, and thus contributes to healthier and more sustainable diets. This paper reviews progress in this area, as well as processing techniques which can be utilised to enhance the sensory characteristics and acceptability of wholegrain cereal products.

\section{Consumer perception of wholesome cereal products}

Foods with health benefits need to have high sensory acceptance in order to be chosen and eaten, and it is of great importance to identify causes of sensory concerns in wholegrain foods. In addition to sensory product properties, extrinsic properties such as packaging, product information, claims etc. ${ }^{6-12}$ Influence the acceptance of a product. Preferences for the sensory attributes found in refined bread are often stated as the reason for the relatively low consumption of whole wheat bread and other wholegrain cereal foods.

Hersleth showed that texture attributes in bread are important for consumers' sensory perception, and Heenan demonstrated that odour and flavour attributes are positive drivers of bread freshness. Bakke and Vickers (2007) conducted an acceptance test including nine different breads chosen to represent comparisons between equivalent refined and whole wheat breads, and concluded that sensory preferences may be a barrier to whole wheat bread consumption, but ingredient 4 or processing modifications can improve liking of such products. Arvola et al. (2007) suggested that making more wholegrain foods available with sensory characteristics comparable to refined grain foods would be a good strategy to promote wholegrain food consumption. Moreover, traditional bread consumption is changing among young consumers from home use towards fast food bread and bread consumed outside home. Thus, there is a current need to develop new appealing product concepts as well as tailored communication strategies focused towards younger consumers. Nudging has recently been studied as a tool to influence healthy food choice by affecting unconscious behavioural processes. Van Kleef provided school children with bread in regular or funny shapes. Their intervention study showed that whole wheat bread consumption doubled when presented as fun-shaped products, demonstrating that visual appeal can stimulate wholegrain consumption.

\section{Flavour- active compounds in wholegrains}

Cereal grains each have their characteristic flavour profiles and precursors, even though the flavour of native, untreated grains is mild and bland. The flavour and texture of cereal foods are mostly formed during processing due to process-induced changes in the grain biopolymers and flavor active compounds. White wheat flour furnishes a small amount of volatile compounds and aroma precursors, and their contribution to bread flavour is estimated to be small. In wholegrain wheat flour, the amount of volatile compounds as well as amino acids is considerably higher. Fermentation and baking are the main sources of flavour of wheat bread, and both steps are essential. Volatile compounds are generated from previous precursors present in ingredients or resulting from enzymatic or mechanical degradations. The most important precursors of the identified compounds are sugars and amino acids. Volatile compounds, such as aldehydes, ketones and alcohols, are perceived as odour, and have traditionally been studied for decades as the only contributors of perceived grain and cereal product flavour causing for example green, sweet and fruity odour notes. For example, volatile compounds in the headspace of bread give information on the freshness of bread. More recently, the role of non-volatile compounds, such as phenolic compounds, amino acids, small peptides, fatty acids and sugars, as flavour- active compounds has been realized. The most dominant and challenging taste caused by some of these is the bitter. The number of other sensory attributes besides bitterness detected as typical of wholegrain, bran and germ is very limited. Bitter flavour of rice correlates positively with dark colour.

Rancidity, as a result of lipid hydrolysis and subsequent fatty acid oxidation, is known to be another contributor to bitterness, and is known to create specific off-flavours. In general, bran and especially 
germ have higher lipid content than endosperm, and are therefore more susceptible to oxidation. If these lipid-rich parts of grain are used, they obviously contribute a lot to the perceived flavour due to oxidation during storage. The role of lipids depends on the variety of grain: in oats lipids are present in the entire grain, in wheat, corn and sorghum they are concentrated in the germ, and lipid oxidation activity is especially high in barley germ and pearl millet.Among wholegrain foods, the role of lipids has been studied most in oats, which contain about $6 \%$ lipids. Depending on processing conditions, lipid hydrolysis may occur by enzymatic or chemical reactions, and subsequent fatty acid oxidation may create off-flavours. Control of free fatty acid formation is thus important with respect to flavour stability of wholegrain foods. Lipid hydrolysis has the most significant impact on flavour stability of wholegrain flour, although some smaller changes may also be caused by protein or carbohydrate degradation During storage, lipolysis takes place, and has a negative impact on the sensory characteristics of flour, perceived as rancid and bitter notes. The shelf-life of whole wheat flour is shorter than that of white flour. Rancidity appears alike more pronounced in wholegrain than refined wheat bread during storage due to lipid oxidation (Table 1).

Table I Examples of typical sensory descriptors of wholegrain and white wheat products

\begin{tabular}{lll}
\hline Wholegrain & Product & Examples of typical sensory attributes \\
\hline Wholegrain wheat & Bread & $\begin{array}{l}\text { Dark colour,'speckled' appearance Coarse, hard texture Nutty odour Bitter/ sour taste } \\
\text { Grain-like, 'seedy' flavour, malted note, musty }\end{array}$ \\
& Biscuit, cracker & Astringent High wheat, toasted and earthy notes \\
White wheat & Bread & Light colour Only slight grain-like flavour or malted note \\
& Biscuit, cracker & Only slight wheat, toasted and earthy notes; strong dairy note \\
\hline
\end{tabular}

Oats is different from other cereal grains due to its high $\beta$-glucan and lipid contents and low starch content. Oats has a positive health image, and it is often used as wholegrain. It is perceived as a tasty cereal giving a nutty flavour, but may have a bitter off-flavour originating from its poor stability, and the tendency to rancidity limits the use of oat. Thus, the inhibition of the lipase activity in oat processing is necessary. High water content and phenolic compounds have been shown to contribute to rancidity and bitterness in oat.

\section{Flavour and texture formation in processing wholegrains and bran}

Good taste, together with other perceived characteristics, is the most important criterion for food choice, and an essential requirement for products to survive on competitive markets. Sensory characteristics of refined and wholegrain products differ in many aspects, and cereal products containing wholegrain or bran face numerous challenges in this respect. As compared to refined products, the main sensory concerns and barriers to overcome in wholegrain and high-fibre foods are flavour, texture and colour.

Flavour is a simultaneous perception of taste, odour and chemical stimuli. The nature and relative amount of volatile and non-volatile flavour-active compounds, their availability to the senses as a function of time, and the mechanism of perception determine flavour perception. Eating is a dynamic process: the profile of compounds in mouth changes as a function of time, when the structure of the food changes during chewing. Flavour perception depends also on chemical and physical binding of the flavour-active compounds within the starch/ protein matrix. Wholegrain products include the outer layers of the grain, which contain several non-volatile, flavouractive substances. Lignocellulosic layers, large particles and insoluble fibre may also cause a hard and gritty texture in a product. DF content of cereals varies considerably, being $11.2 \%$ in wholewheat flour, $10.6 \%$ in naked oat, $18.5-23.4 \%$ in hulled oat, $17.7-19.3 \%$ in wholegrain rye, $20-24 \%$ in barley and $0.7-19.2 \%$ in rice. High DF content decreases loaf volume, increases hardness of crumb, decreases crispiness, changes colour and causes aftertaste in baked products. The colour of bran is often dark, affecting food acceptance. Non-appetising colour, such as a dark colour of rye, greyish colour of barleyor 11 dark colours of rice and sorghum are suggested to be caused by polyphenols, and often to be associated with bitter or astringent taste. Colour and texture may be even more important than flavour for the acceptance by consumers used to eat refined cereal foods.

At high temperatures, free amino acids or small peptides together with free sugars are especially important flavour precursors and form volatile flavour-active compounds, such as heterocyclic pyrazines, pyrroles, furans, and sulphur-containing compounds. These compounds are especially abundant in high-temperature, lowmoisture extruded oats, and the products are described as roasted or toasted, caramelized and somewhat sweet, or nutty in flavor. contrast, proteolytic reactions release peptides which are perceived as less appealing. Enzymatic reactions release compounds from the matrix, or hydrolyse or oxidise the compounds, and have in that way impact on flavour formation.

The ash content of flour, indicating presence of outer grain layers and hence more nutrients and precursors for fermentation, has been reported to be a major factor in determining the intensity of the sensory attributes of sourdough bread, the bread being most preferred if flour with low or medium levels of ash was used. Utilisation of wholegrain flour in wheat bread increased intensity of flavour, aftertaste and also pungent flavour of bread. Addition of wheat bran (15\% supplementation level) to wheat bread similarly increased flavour intensity, pungent flavour, aftertaste and coarse mouthfeel in sourdough baking. Already 5\% bran addition to wheat flour increased the amount of free amino acids, total phenols, dietary fibre, and phytic acid and antioxidant activities of dough compared to white wheat flour, enhancing potentially bitter and pungent flavour notes. In addition, sourdough fermentation still increased the amount of these nutrients in baked breads, and concurrently enhanced the intensities of sensory attributes.

\section{Approaches to improve sensory attributes of wholegrain and bran - containing cereal foods}

The flavour and texture of cereal foods are formed during processing, and the choice of raw materials and ingredients is the first step when considering sensory design. Processing of cereal foods is often made by combination of several unit operations. Technologies used to add more of the grain in cereal foods were recently reviewed 
by Poutanen. In bread baking, for example, means to modulate product quality include selection of the flour type and other ingredients, and improving the gluten network either by using added gluten or dough improvers, or by processing techniques and parameters, such as sourdough fermentation, proofing time and temperatures etc. Use of enzymes is one potential tool for modifying the flavour and especially texture of bakery products. Bioprocessing provides new tools for flavour and texture design of palatable, health-beneficial breakfast and snack applications from cereals by removing unwanted flavours or by enhancing desired flavours. Basic knowledge models will also provide a good basis for design of structure at various levels, provided they also are applied to systems high in bran and wholegrain

\section{Breeding or choice of raw materials}

The colour of the pericarp of wheat kernels can vary widely, from white to golden yellow, red and even purple. Most abundant bread making wheats today are red, resulting in distinct red-brown coloured bran particles in wholegrain and overall dark-brown bread. Dark bran usually contains tannins and phenolic acids, which give whole wheat flour the slightly bitter taste, whereas white whole wheat has a mild, sweet and slightly nutty flavour without a trace of bitterness. The use of white wheat varieties is reported to provide products with a lighter colour, but also milder flavor When the flavour profile of different varieties of wheat was compared, the ancient species Emmer, Einkorn and Spelt as well as some old varieties had milder taste and aroma than the modern varieties in the group tested. Comprehensive studies about the genetic variance in flavour profile of grains are not, however, available. Sorghum cultivars with high levels of condensed tannins have major agronomic advantages, and food products made thereof have a high antioxidant capacity. However, cooked wholegrain kernels from tannin sorghums having a relatively soft endosperm texture were perceived as dark, hard, chewy, bitter and astringent, while tannin-free sorghums having relatively hard endosperm texture were perceived as soft, sweet and having a maize-like flavour. Kobue-Lekalake showed that selection of sorghum cultivars containing low concentration of tannins may both promote health and ensure preference by consumers There seems to be tannin- containing sorghums that are palatable, even to bitter-sensitive consumers, because the tannin levels are below a detection threshold. Such tannin sorghums could be used to produce high antioxidant activity foods that are acceptable to consumers.

\section{Milling and fractionation}

Enzymes, such as peroxidases, polyphenoloxidases and amylases of the aleurone layer are liberated from tissues during milling, leading to off-flavour development, pigment darkening, and starch breakdown. The type of milling technique has been shown to have a greater impact on the sensory quality of wholegrain wheat bread than the baking technique: bread baked with roller-milled wheat was described as being sweet, juicy, and compact, with a small slice area, whereas bread baked with stone milled wheat was salty, deformed, and roasted. Similarly, Kebakile and Khumalo showed that different milling methods greatly influenced the sensory properties, 24 in particular texture but also visual properties of sorghum and maize porridges. Efficient separation of the pericarp from the starchy endosperm depends on the structural integrity of the pericarp and the milling process used. For sorghum, quantity of specks and porridge colour are associated with pigmentation of testa (if present) and the pericarp properties of the sorghum kernel. Removal of the very outer layers of the grain in milling (peeling) might offer one tool for improving, in addition to safety, also the texture and flavour of the resulting products. Flavour components are unevenly distributed in the kernel and thus choice of suitable milling fractions is one option for production of flours rich in e.g. DF but mild in taste. Between the mild-tasting inner endospermic part of rye grain and the bittertasting outermost bran fraction (pericarp), a rather mildtasting fraction (aleurone) was detected still high in bioactive compounds, such as alk(en)ylresorcinols and phenolic acids. Polishing or slight peeling of the grain to remove the surface layers of the grain may also influence processing behaviour by removing microbially derived enzymes and lignocellulosic hard structures covering the grain, while leaving most of the DF and associated compounds in the raw material. Mechanical peeling of rye bran used at $20 \%$ addition level in wheat bread decreased significantly the flavour and colour intensity, but concurrently some of the bioactive compounds located in bran were lost (Heiniö, unpublished results). Fermentation of bran produced from peeled grains improved the flavour of bran-enriched breads.

\section{Bioprocessing}

Bioprocessing includes use of microbes, enzymes and germination of ingredients at different processing steps. Sourdough fermentation has traditionally been used in processing of wholegrain flour, and is known to influence nutritional and sensory properties as well as stability. The reasons for flavour modifications of wholegrain flour are based mainly on: a) 26 intensive proteolysis producing amino acids in wholegrain sourdoughs due the activation of cereal proteases at a low $\mathrm{pH}$ during long fermentations, $\mathrm{b}$ ) release of phenolic compounds from wholegrain flour during fermentation, and c) more intensive acidification occurring in wholegrain flour as compared to refined flour. Biochemical changes during fermentation are responsible for both desired and undesired modifications in subsequent bread flavour. Using sourdough as a flavour improver requires therefore carefully optimised fermentation conditions providing moderate acidity and enhanced level of amino acids, as well as enhanced level of certain volatile compounds for producing a balanced bread sensory profile. Furthermore, strain-specific influences on bread flavour are evident. In general, lactic acid bacteria (LAB) -containing sourdoughs modify bread flavour more effectively; producing both desired and undesired sensory attributes. Sourdoughs containing yeast modify bread flavour less effectively, and roasted crust flavour cannot be enhanced at all with yeasted sourdoughs. Diminished roasted flavour due to the use of yeasted preferment has been reported by Zehentbauer and Grosch. Yeasted preferment has been reported to either improve or have no effect on bread flavour, opposite results being most likely due to different processing conditions of pre-fermentation and the variation of measured sensory attributes in different studies. Moderate level of acidity with enhanced levels of amino acids has been obtained by using e.g Lactobacillus brevis as a starter with wholegrain flour for 20 hours at $24^{\circ} \mathrm{C}$, delivering improved flavour. High temperature in combination with long fermentation time is a key factor for intensive acidification in $\mathrm{LAB}$ fermented sourdoughs but less important for intensive proteolysis or formation of volatile compounds, or for sensory attributes of subsequent bread. Thus, use of lower temperature allows controlling acidity development and at the same time the high enzyme activity of wholegrain flour and long fermentation time promotes proteolysis.

\section{Use of ingredients to mask bitterness of wholegrain}

Bitterness is a common sensory concern of plant derived foods. Bitter-tasting compounds are therefore often removed from plant foods through breeding or debittering processes. Debittering methods have been suggested for other plant foods, but it is more feasible in liquid food products such as beverages. In wholegrain, the cereal and DF matrices themselves might act as adsorbers of flavouractive compounds, the intensity of which may thus be modulated 
by processing. Better understanding of taste receptors on the human tongue may lead to28 further innovations in flavour-masking systems. In addition to masking, the perception of bitterness can be reduced by blocking the bitter taste receptor hTAS2R39. Three 6- methoxyflavanones were shown to reduce hTAS2R39 activation by epicatechin gallate (ECG). These bitter receptor blockers were characterized as reversible antagonists. Furthermore, complexation of epigallocatechin gallate (EGCG) with food proteins reduced hTAS2R39 activation. A trained sensory panel confirmed reduced bitterness perception. Sweeteners are often used as flavour-masking agents in whole grain products, and e.g. breakfast cereals made of wheat bran contain over $10 \%$ sugar. Many bakers are turning to honey to build on its ability to mask off-flavours of wholegrain wheat. In addition to sweeteners, also salt may offer an option in masking the bitter taste. The bitterness and astringency of rice have shown to be significantly reduced by adding salt or salt with oil to water in cooking. This could also be the case in mixed meals, like pasta with cheese souce, as sodium cations have been shown to mask bitter compounds. Masking bitter off-notes in wholegrain products may be complex in terms of understanding the difference between singular and multi-dimensional off-notes which require masking. Three approaches in masking off-notes in wholegrain products have been suggested: a) adding lubricity via saliva-stimulating agents, b) mouthfeel-enhancers and fat-simulators; masking the bitter and oxidized notes often present in whole grain products, particularly by enhancing sweetness, and c) using delivery systems to prevent flavor compounds forming complexes with fibre. Inferior texture formation properties of wholegrain flour can be compensated by adding standard baking aids such as gluten, emulsifiers, enzymes and by optimisation of the baking process. A widely used way to reduce the negative effects of bran is by compensations made in the bread formula by addition of vital gluten, or the use of surfactants

\section{Conclusion}

Wholegrain and bran are among the health beneficial ingredients which consumption should be increased. They contain flavour-active compounds and flavour precursors as well as resistant cell wall structures which may during processing cause changes in flavour and texture attributes reducing their acceptance of wholegrain foods by consumers. Considering the major role of cereal foods in daily diet and nutrition, it is surprising that the scientific literature of factors influencing sensory quality of wholegrain foods is rather limited. It is also interesting that actually many food grains, with the exception of wheat, are traditionally used as wholegrain. The concern about increasing the use of whole vs. refined grain foods has thus been largest in case of wheat products. Bitterness is the key sensory attribute known to restrict the use of many plant-derived foods. In wholegrain foods, different chemical constituents contribute to bitterness: e.g. certain phenolic compounds, peptides, and Maillard reaction compounds. While their intensities can be controlled by careful choice of raw materials, processing is the most important step to control bitterness. As the flavour stimuli are created in bitter taste receptors, controlling their activation is one key area for future research. Binding of bittertasting compounds in the cereal food matrix, e.g. through the use of natural or added adsorbents, is another option. Control of endogenous enzymes in the raw material is also essential, as formation of peptides and oxidation of lipids may add off-flavours to wholegrain foods.
Enzymes, both those in grains themselves and added as processing aids, are on the other hand a good tool especially in design of texture but also flavour of wholegrain foods. This is one of the reasons for the traditional sourdough processing in baking of wholegrain breads, a technique also having clear future potential.

\section{Acknowledgments}

None.

\section{Conflicts of interest}

There is no conflict of interest.

\section{Funding}

None.

\section{References}

1. Aboubacar A, Kirleis A, Oumarou M. Important sensory attributes affecting consumer acceptance of sorghum porridge in West Africa as related to quality tests. Journal of Cereal Science. 1999;30(3):217-225.

2. Adams J, Engstrom A. Dietary intake of whole grain vs. recommendations. Cereal Foods World. 2000;45(2):75-78.

3. Alam SA, Järvinen $\mathrm{J}$, Kirjoranta $\mathrm{S}$, et al. Influence of particle size reduction on structural and mechanical properties of extruded rye bran. Food and Bioprocess Technology. 2014;7(7):2121-2133.

4. Andersson AAM, Gimberg L, Åman P, et al. Recent findings on certain bioactive compounds in whole grain wheat and rye. Journal of Cereal Science. 2014;59:294-311.

5. Aravind N, Sissons M, Fellows CM. Effect of soluble fibre (guar gum and carboxymethylcellulose) addition on technological, sensory and structural properties of durum wheat spaghetti. Food Chemistry. 2012;131(3):893900.

6. Arvola A, Lähteenmäki L, Dean M, et al. Consumers' beliefs about whole and refined grain products in the UK, Italy and Finland. Journal of Cereal Science. 2007;46(3):197-206.

7. Bredie WLP, Mottram DS, Hassell GM, et al. Sensory characterisation of the aromas generated in extruded maize and wheat flour. Journal of cereal Science. 1998;28(1):97-106.

8. Brijs K, Bleukx W, Delcour JA. Proteolytic activities in dormant rye (Secale cereale L.) grain. Journal of Agricultural and Food Chemistry. 1999;7(9):3572-3578.

9. Bustos MC, Perez GT, León AE. Sensory and nutritional attributes of fibre-enriched pasta. LWT Food Science and Technology. 2011;44(6):1429-1434. ;

10. Callejo MJ. Present situation on the descriptive sensory analysis of bread. Journal of Sensory Studies. 201126(4):255-268.

11. Callejo MJ, Vargas-Kostiuk M, Rodríguez-Quijano M. Selection, training and validation process of a sensory panel for bread analysis: Influence of cultivar on the quality of breads made from common wheat and spelt wheat. Journal of Cereal Science. 2015;61:55-62.

12. María Jesús Callejo, María-Eugenia Vargas-Kostiuk, Marta RodríguezQuijano. Selection, training and validation process of a sensory panel for bread analysis: Influence of cultivar on the quality of breads made from common wheat and spelt wheat. Journal of Cereal Science. 2015;61(2015):55-62. 